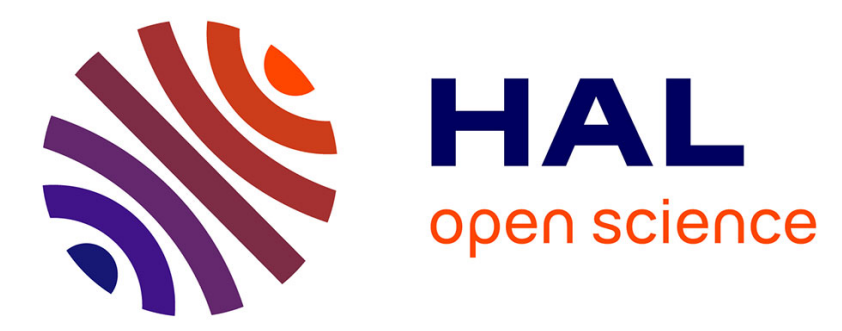

\title{
Acoustical spring effect in a compliant cavity
}

Bruno Issenmann, A. Auberon, Régis Wunenburger, Jean-Pierre Delville

\section{To cite this version:}

Bruno Issenmann, A. Auberon, Régis Wunenburger, Jean-Pierre Delville. Acoustical spring effect in a compliant cavity. European Physical Journal E: Soft matter and biological physics, 2013, 36 (4), pp.39 10.1140/epje/i2013-13039-6 . hal-00825443

\section{HAL Id: hal-00825443 \\ https://hal.science/hal-00825443}

Submitted on 6 Mar 2018

HAL is a multi-disciplinary open access archive for the deposit and dissemination of scientific research documents, whether they are published or not. The documents may come from teaching and research institutions in France or abroad, or from public or private research centers.
L'archive ouverte pluridisciplinaire HAL, est destinée au dépôt et à la diffusion de documents scientifiques de niveau recherche, publiés ou non, émanant des établissements d'enseignement et de recherche français ou étrangers, des laboratoires publics ou privés.

\section{다)(1) $\$$}

Distributed under a Creative Commons Attribution - NonCommercial| 4.0 International 


\title{
Acoustical spring effect in a compliant cavity
}

\author{
B. Issenmann ${ }^{1,2,3,4, a}$, A. Auberon ${ }^{1,2}$, R. Wunenburger ${ }^{1,2,5,6}$, and J.-P. Delville ${ }^{1,2}$ \\ 1 Université Bordeaux, LOMA, UMR 5798, F-33405 Talence, France \\ 2 CNRS, LOMA, UMR 5798, F-33405 Talence, France \\ 3 Université Lyon 1, Institut Lumière Matière, UMR5306, F-69622 Villeurbanne Cedex, France \\ 4 CNRS, Institut Lumière Matière, UMR5306, F-69622 Villeurbanne Cedex, France \\ 5 UPMC Université Paris 06, UMR 7190, Institut Jean Le Rond d'Alembert, F-75005 Paris, France \\ ${ }^{6}$ CNRS, UMR 7190, Institut Jean Le Rond d'Alembert, F-75005 Paris, France
}

\begin{abstract}
We report on the first dynamic study of acoustical spring effect in a compliant cavity formed between a spherical ultrasonic transducer immersed in water and the free liquid surface located at its focus. As its optical analog, this effect is due to the mutual feedback between the cavity length $L$ and the large acoustical power stored inside the cavity, here through acoustic radiation pressure. We use surface waves to investigate the acoustical spring effect. The amplitude of surface waves above the cavity is observed to vary with the slope of variation of the $L$-dependent acoustic radiation pressure exerted on the liquid surface, i.e. with the acoustic spring stiffness. The observed simultaneous back-scattering of these surface waves demonstrates that the surface response to the cavity length variations results mainly in an added stiffness, i.e., in an increase of the real part of the surface impedance above the cavity. Finally, when the liquid surface is located out of the focal plane, spontaneous surface oscillations are reproducibly observed, which may be due to a parametric instability.
\end{abstract}

\section{Introduction}

The understanding and the control of the optomechanics of deformable optical cavities, i.e., of the mutual feedback between the dynamics of the cavity and the circulating light field, is the cornerstone of several challenging realizations such as detecting gravitational waves [1-4], controlling atomic force microscopes [5-8], or reaching the quantum limit of accuracy for position measurements [9-11].

Consider an optical cavity in which one of the mirrors is mounted on a spring that is so compliant that it can be displaced by the radiation pressure of the light circulating within the cavity. Since the optical power stored in the cavity depends in turn on the cavity length, that is to say on the position of the mobile mirror, a mutual parametric feedback exists between the cavity and the light stored within it. As a result, various phenomena can be observed, depending on the characteristic features of the optomechanical feedback.

An in-phase dependence on the mirror position of the force exerted by light on the mirror results in an effective stiffness ("optical spring effect") which is added to the spring stiffness and modifies the resonance frequency of the mirror $[6,8,12-14]$. If the net mirror stiffness is negative, bistability occurs $[8,15,16]$.

\footnotetext{
a e-mail: bruno.issenmann@univ-lyon1.fr
}

When the optical finesse of the cavity is so high that the characteristic timescale of leakage of the cavity is comparable to the period of the free oscillations of the mirror, the circulating light intensity adapts to the cavity length variations with a noticeable delay (time-delayed feedback). The resulting force exerted by light on the mirror varies out of phase with respect to the mirror position: a component proportional to the mirror velocity arises. Depending on its sign, the moving mirror experiences either damping or anti-damping $[17,18]$. Anti-damping increases the quality factor of resonators and triggers a parametric, oscillatory instability when it overcomes the mechanical damping of the resonator. For large optical intensities, all these combined effects result in a complex dynamics [19-21] and eventually chaos [22].

Given the recent demonstration that acoustic radiation pressure can actually be used to actuate an AFM cantilever in liquid phase on the one hand [23,24] and the large variety of applications of the optomechanical coupling on the other hand, it is appealing to determine whether or not such phenomena can occur when acoustic waves are confined in a compliant cavity. More generally, since the radiation pressure associated to an electromagnetic, or acoustic, wave of intensity $I$ scales as $I / c$, where $c$ is the wave velocity, acousto-mechanical effects are expected to be typically $10^{5}$ times larger than optomechani- 
cal effects for a given intensity. This allows to consider the application of the acousto-mechanical couplings to macroscopic and stiffer systems.

\section{Principles of the experiments}

We have previously evidenced the bistable behavior of a compliant cavity induced by acoustic radiation pressure [25]. In this article, we report on the first dynamic study of the acoustical analog of the optical spring effect. The experimental scheme is the following. As sketched in fig. $1 \mathrm{~b}$, a cavity is formed between the partially reflecting, hemispherical surface of an ultrasonic transducer immersed in water and emitting vertically, and the perfectly reflecting, water free surface located in the transducer focal plane. The transducer emitting a continuous sine wave, a steady bell-shaped deformation (hump), shown in fig. 1d, forms at the free surface as the result of the acoustic radiation pressure exerted on it by the stationary acoustic field set up in the cavity. Our previous study has shown that, as far as the reflection of the acoustic wave is concerned, the surface hump can be satisfactorily described as a plane mirror located at the hump tip [25]. In order to evidence the change of stiffness of the liquid surface, we trigger a plane, progressive surface wave using a vibrating blade, see fig. 1a and b. This wave propagates toward the hump. First, by comparing its amplitude on the hump with its amplitude at the same location in the absence of hump, we show that the amplitude of the surface wave is reduced on the hump proportionally to the slope of the variation of the hump height-dependent radiation pressure exerted on the hump tip (acoustic spring constant). Then, by detecting a stationary surface wave upstream from the hump resulting from the interference between the incident surface wave and the wave which is backscattered by the hump, we show that the surface response to the cavity length variations mainly results in an increase of the real part of the surface impedance above the cavity, i.e. in an added stiffness. Finally, we report on spontaneous surface oscillations which are reproducibly observed when the liquid surface is located out of the focal plane at rest, and we discuss their possible origin.

\section{Experimental determination of the finesse of the acoustic cavity}

Each experiment is performed using the following setup and protocol. A spherical Imasonic ultrasonic transducer (focal length $d_{F}=38 \mathrm{~mm}$, aperture $N A=1, f=2.25 \mathrm{MHz}$ central frequency, $600 \mathrm{kHz}$ bandwidth, focal zone length $d_{z}=3.2 \mathrm{~mm}, 5 \mathrm{~s}$ maximal sonication time) is immersed in pure, degassed water contained in a transparent tank, see fig. 1a. It is fed by a power amplifier supplied by a PCcontrolled waveform generator emitting a sine signal with fixed frequency $f$ and slowly varying amplitude $V(t) . V(t)$ is extracted using an amplitude demodulator and sent to a digital oscilloscope. The surface hump is illuminated at grazing incidence by a parallel beam of white light. The

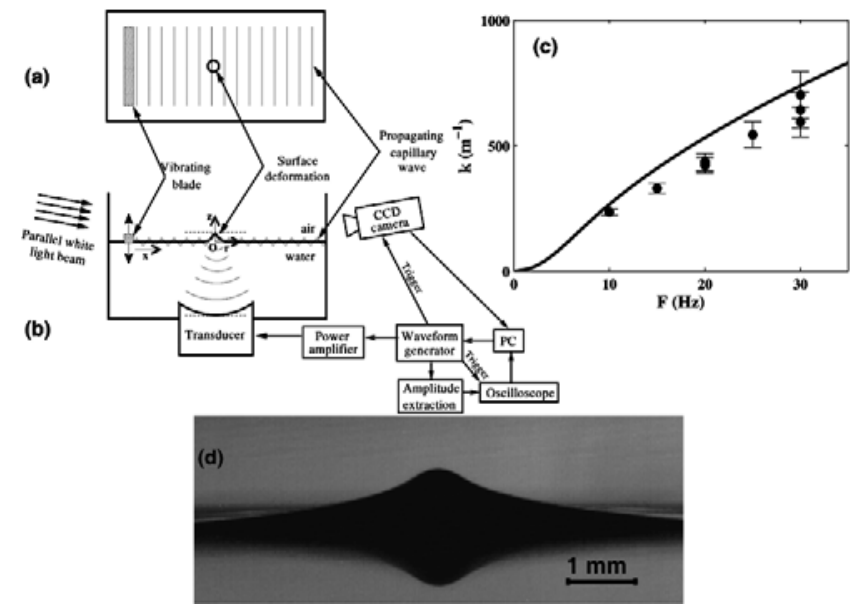

Fig. 1. Experimental setup top view (a) and side view (b) (c) Dispersion relation of gravito-capillary waves. Symbols: Measurements. Plain curve: theoretical dispersion relation in deep water: $\Omega=\sqrt{g K+\sigma K^{3} / \rho}$. (d) Picture of the steady surface hump induced by acoustic radiation pressure in the absence of the incident surface wave (the hump pointing downward is the reflection of the real surface hump on the liquid surface).

pictures of the surface hump are captured at $1000 \mathrm{fps}$ using a high-speed CCD camera triggered by the waveform generator. In order to evidence the hysteresis associated to the bistable behavior of the compliant cavity, we ramp $V^{2}$ linearly with time up and down within $5 \mathrm{~s}$ and simultaneously measure the variations of the height $h_{s}$ of the surface hump as a function of time. Since the measured characteristic timescale of relaxation of the surface hump is a ten of milliseconds, in the absence of surface waves the deformation is assumed to evolve adiabatically during the voltage ramps. The $V(t)$ and $h_{s}(t)$ signals acquired in the absence of surface waves are plotted in fig. 2 . The hysteretic behavior of $h_{s}(V)$, which results from the strong acousto-mechanical coupling, is evidenced in the inset of fig. 2 .

In ref. [25], we have shown that the combination of a one-dimensional model of Fabry-Pérot cavity and of a prediction of the small-amplitude, axisymmetric, static surface deformation induced by the radiation pressure exerted by the acoustic field set up in the cavity satisfactorily fits with no adjustable parameter the measured dependence of the hump height $h_{s}$ versus the pressure oscillation amplitude at focus $P_{e} \propto V$ of the wave emitted by the transducer and injected into the cavity. Since in this study we aim at characterizing the acoustic spring effect, we need to accurately describe the $h_{s}$-dependence of the radiation pressure $\Pi=P_{i}^{2} /\left(\rho c^{2}\right)$ exerted on the liquid surface, where $P_{i}$ is the pressure oscillation amplitude of the wave incident on the surface (i.e. of the injected wave combined with its multiple reflections incident on the surface), $\rho=1000 \mathrm{~kg} \mathrm{~m}^{-3}$ the water density and $c=1.5 \mathrm{~km} \mathrm{~s}^{-1}$ the wave velocity in water. This is done by deducing $P_{i}^{2}$ from the measured $h_{s}$ using the model of small-amplitude static 


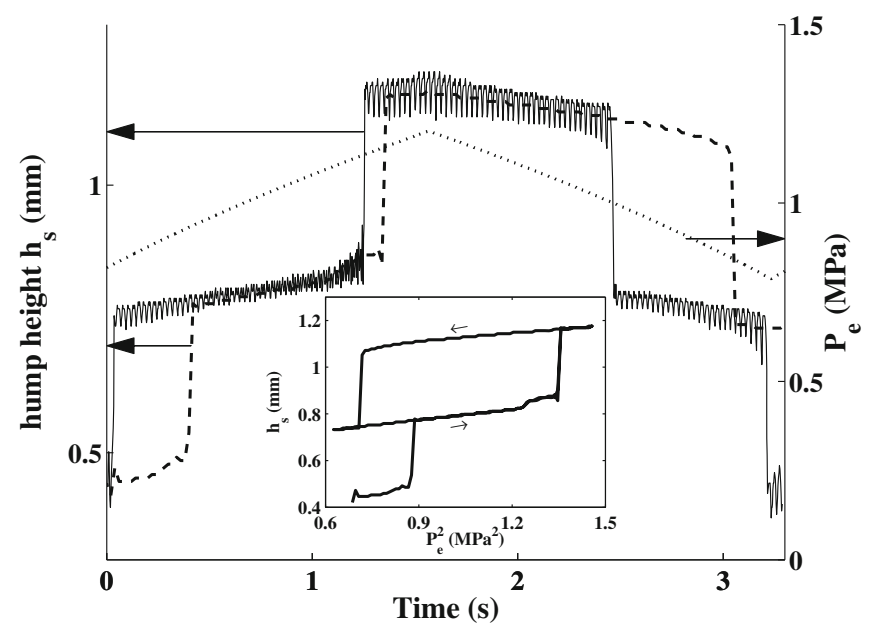

Fig. 2. Dotted curve: predicted variation of the pressure oscillation amplitude at focus $P_{e}$ associated to the acoustic wave emitted by the transducer during an up-and-down $V$-ramp (pressure scale on the right-hand vertical axis). Corresponding variation of the hump height in the absence of incident surface wave $h_{s}(t)$ (dashed, bold curve) and in the presence of an incident surface wave which induces oscillations of the hump height $\delta h(t)$ (solid curve) (height scale on the left-hand vertical axis). Inset: experimental hysteresis cycle stridden by the hump height $h_{s}$ as function of $P_{e}^{2}$ when the voltage driving the transducer $V$ is ramped up and down in the absence of surface wave.

surface deformation [25]

$$
P_{i}^{2}=h_{s}\left[\frac{w^{2}}{8 \sigma \rho c^{2}} \exp \left(\frac{\rho g w^{2}}{8 \sigma}\right) E_{1}\left(\frac{\rho g w^{2}}{8 \sigma}\right)\right]^{-1},
$$

where $w=0.86 \lambda$ is the characteristic width of the radial distribution of the pressure oscillation amplitude of the incident acoustic beam at focus $P_{i}(r)=P_{i} \exp \left(-r^{2} / w^{2}\right)$ $(r$ is the radial coordinate associated to transducer vertical $z$ axis), $\lambda=670 \mu \mathrm{m}$ the acoustic wavelength at frequency $f=2.25 \mathrm{MHz}, \sigma=72 \mathrm{mN} \mathrm{m}^{-1}$ the water surface tension, $g=9.8 \mathrm{~m} \mathrm{~s}^{-1}$ gravity acceleration, and $E_{1}$ the 1-argument exponential function [26]. $P_{e}(V)$ is determined from independent pressure measurements in bulk water using a hydrophone. The resulting experimental determination of the $h_{s}$-dependence of $\left(P_{i} / P_{e}\right)^{2}$ is shown in fig. 3. Only the right-hand side of the resonance peaks could be recovered since almost their whole left-hand side corresponds to unstable equilibrium states of the acousto-mechanical system. The model of ref. [25]

$$
\left(\frac{P_{i}}{P_{e}}\right)^{2}=\left(1-R R^{\prime}(h) \exp \left(i \frac{4 \pi\left(L_{0}+h_{s}\right)}{\lambda}\right)\right)^{-2},
$$

is also shown in fig. 3 , where $L_{0}$ is the cavity length when the liquid surface is flat, $R=-0.38$ the pressure reflection coefficient of the transducer surface, $-1 \leq R^{\prime}\left(h_{s}\right)<0$ a $h_{s}$-dependent effective pressure reflection coefficient of the liquid surface that quantifies using a ray acoustics approach the decrease of the finesse of the acoustic cavity due

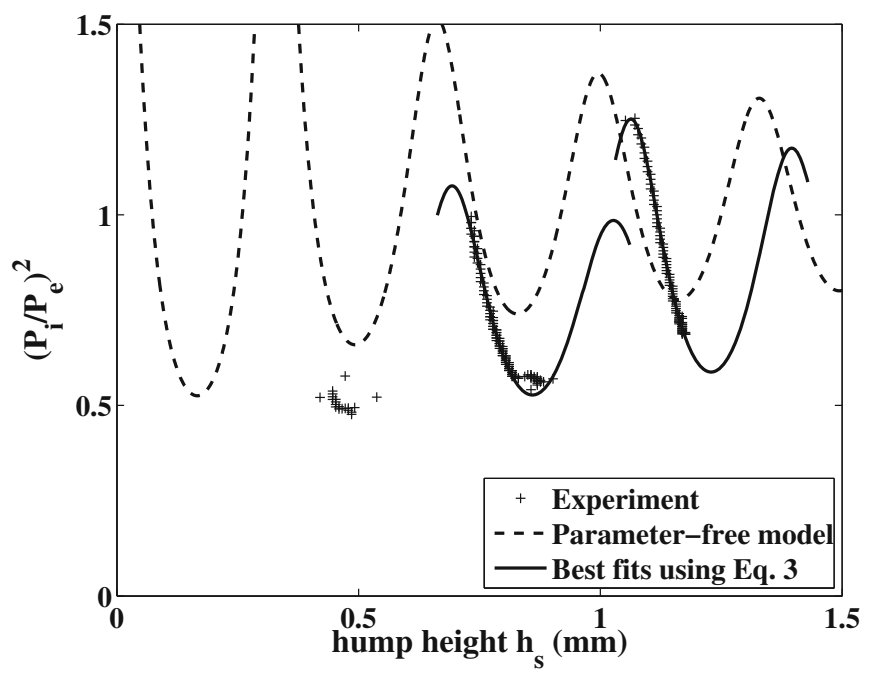

Fig. 3. Symbols: square of the ratio between the pressure amplitude $P_{i}$ of the incident beam and the pressure amplitude $P_{e}$ of the beam emitted by the transducer and injected into the cavity as a function of the height of the steady hump $h_{s} . P_{i}$ is experimentally deduced from the deformation height $h_{s}$ using eq. (1). $P_{e}$ is computed from the electric voltage at the transducer. Dashed curve: theoretical prediction using eq. (2) with no adjustable parameter. Solid curves: theoretical prediction using eq. (3).

to the defocusing of the reflected beam by the curved mirror [25]. Although satisfactory, the agreement between the measured and the predicted $\left(P_{i} / P_{e}\right)^{2}(h)$ variations shown in fig. 3 is not accurate enough to quantitatively model the actual $\Pi\left(h_{s}\right)$ variations. In order to get an analytical and accurate representation of the experimental $\Pi\left(h_{s}\right)$ variations, we have closely fitted them around each resonance peak with an effective model of resonance

$$
\begin{aligned}
& \left(\frac{P_{i}}{P_{e}}\right)^{2}= \\
& {\left[\left(1-R^{*} R^{\prime}\left(h_{s}\right) \exp \left(i \frac{4 \pi\left(L_{0}+h_{s}+\Delta h\right)}{\lambda}\right)\right)\right]^{-2}+C,}
\end{aligned}
$$

where $R^{*}, \Delta h$ and $C$ are three fitting parameters. As shown in fig. 3 , the corresponding $\left(P_{i} / P_{e}\right)^{2}\left(h_{s}\right)$ variations calculated using eq. (3) accurately describe the experimental data and can thus be confidently derived in order to evaluate $\left(\frac{\partial \Pi}{\partial h}\right)_{P_{e}}$.

\section{Principle of measurement of the acoustic spring effect}

We aim at evidencing the variation of the stiffness of the compliant acoustic mirror formed by the hump by detecting the change of the surface impedance on the hump through its effect on the propagation of surface waves. The propagation of surface waves at a liquid free surface is indeed the result of inertia and of the intrinsic causes of surface stiffness that are gravity and surface tension. 
We expect that the mutual feedback between the acoustic cavity and the hump height results in a variation of the effective stiffness of the liquid surface, hence of the surface impedance on the hump. In order to quantify this effect, we consider a plane, progressive, small-amplitude, harmonic surface wave of pulsation $\Omega$ and wave number $K$ propagating without attenuation toward the hump, see fig. 1a. In the absence of incident surface wave, the steady hump shape $h_{s}(r)$ satisfies the static equilibrium equation $\rho g h_{s}(r)-\sigma \kappa_{s}(r)=\Pi\left(r, h_{s}(r)\right)$, where $\kappa_{s}(r)$ is the surface curvature and $\Pi\left(r, h_{s}(r)\right)$ the acoustic radiation pressure field exerted on the liquid surface. Considering small-amplitude surface height perturbations $\delta h$ of this equilibrium state, linearizing the radiation pressure variations associated to the cavity length variations as $\Pi\left(r, h_{s}+\delta h(r, t)\right)=\Pi\left(r, h_{s}(r)\right)+\left.\frac{\partial \Pi}{\partial h}\right|_{P_{e}}\left(r, h_{s}(r)\right) \delta h(r, t)$, and considering that these radiation pressure variations follow instantaneously the surface height variations, leads to the same set of equations as those governing the standard propagation of surface waves on a flat liquid surface except that the restoring hydrostatic pressure term $\rho g \delta h$ is changed to $\left[\rho g-\left.\frac{\partial \Pi}{\partial h}\right|_{P_{e}}\left(r, h_{s}(r)\right)\right] \delta h$. Assuming that the wavelength of the surface wave $\Lambda=2 \pi / K$ is large compared to the characteristic diameter of the hump, $\Lambda \gg w$, the hump can be considered as a sub-wavelength scatterer that locally increases the surface stiffness by the typical amount $-\left.\frac{\partial \Pi}{\partial h}\right|_{P_{e}}\left(h_{s}\right)$, with $h_{s}=h_{s}(0)$. Away from the hump the surface height perturbation can be written as $\delta h_{i}(x, t)=H_{i} \cos (\Omega t-K x)$, where $H_{i}$ is the amplitude of the incident surface wave. The associated propagating perturbation of the liquid pressure below the surface is $\delta P(x, t)=\left(\sigma K^{2}+\rho g\right) \delta h_{i}(x, t)$. Assuming that the intensity of the surface wave scattered by the hump is small compared to the incident intensity, as experimentally confirmed later, this pressure perturbation attains the hump tip where the surface stiffness is modified by the acoustic spring effect. Consequently, this incident pressure perturbation induces a variation $\delta h=H \cos \Omega t$ of the height of the hump that satisfies $\delta P=\left(\sigma K^{2}+\rho g-\left.\frac{\partial \Pi}{\partial h}\right|_{P_{e}}\left(h_{s}\right)\right) \delta h$. One finally gets

$$
\frac{H_{i}}{H}=1-\frac{\left.\frac{\partial \Pi}{\partial h}\right|_{P_{e}}\left(h_{s}\right)}{\sigma K^{2}+\rho g} .
$$

$H_{i} / H$ is thus predicted to vary linearly with the acoustic spring stiffness. Since in our experiment $\left.\frac{\partial \Pi}{\partial h}\right|_{P_{e}}\left(h_{s}\right)<0$ (see fig. 3), one expects to observe only a reduction of the amplitude of the surface wave on the hump tip $\left(H_{i} / H>1\right)$. Moreover, since the dispersion equation of surface waves $\Omega^{2}=g K+\frac{\sigma}{\rho} K^{3}$ implies that $K$ increases monotonously with $\Omega$, for a given value of $\left.\frac{\partial \Pi}{\partial h}\right|_{P_{e}}\left(h_{s}\right)$, $H_{i} / H$ is predicted to decrease when $\Omega$ is increased. To conclude this section, we predict that the comparison between the amplitude of surface waves on the hump and away from it can be used to quantitatively evidence the acoustic spring effect.

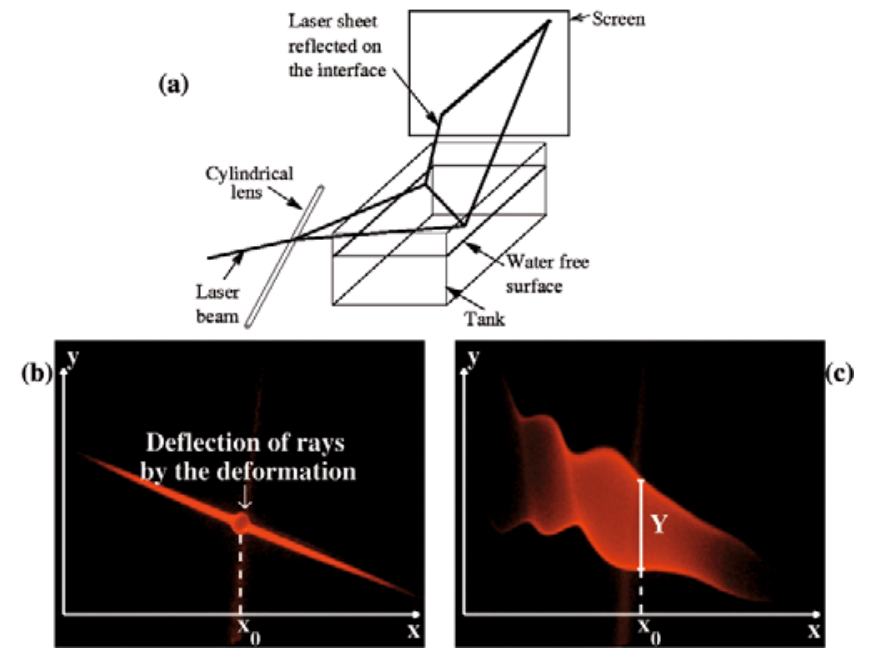

Fig. 4. (a) Setup used to measure the amplitude of the incident surface wave. (b) Picture of the reflected light sheet on the screen in the absence of incident plane wave and in the presence of a small hump. (c) Picture of the broadened light line in the absence of hump and in the presence of an incident plane wave (oscillations of the line broadness at the left of the picture are near field effects close to the vibrating blade).

\section{Experimental evidence of the acoustic spring effect}

The amplitude $H_{i}$ of the plane, incident wave is measured using the setup shown in fig. 4a in the following manner. A plane laser sheet impinges on the surface of water such that it illuminates the hump. This sheet is reflected by the water surface towards a vertical screen. A picture of the reflected light sheet in the absence of incident plane wave and in the presence of a small hump, shown in fig. $4 \mathrm{~b}$, is used to determine the abscissa of the hump imprint on the screen $x_{0}$. When the blade vibrating at frequency $F=\Omega / 2 \pi=30-60 \mathrm{~Hz}$ emits the surface wave $(\Lambda=5-10 \mathrm{~mm})$, due to the retinal remanence, the projection of the reflected light sheet on the screen is seen to broaden proportionally to the wave amplitude. On the one hand, the width $Y$ of the broadened light line, shown in fig. $4 \mathrm{c}$, is measured in the absence of hump at $x_{0}$. The surface wave amplitude $H_{i}$ at the hump location in the absence of hump is deduced from $Y$ using the geometrical relation $H_{i}=\frac{\Lambda Y}{8 \pi d\left(1+\tan ^{2} \alpha\right)}$, where $d$ is the horizontal distance between the hump and the screen and $\alpha$ the incident angle of the laser sheet on the liquid surface.

On the other hand, the amplitude $H$ of the oscillations of the height of the hump is directly measured on the pictures of the oscillating hump acquired at $1000 \mathrm{fps}$ during an up-and-down $V$-ramp. The value of $H_{i}$, typically $500 \mu \mathrm{m}$, is chosen so as to measure $H$ with at least a 10 pixels resolution. The $h(t)$ signal acquired in the presence of an incident surface wave is shown in fig. 2. As expected, $h(t)$ is the superposition of the quasi-steady hump height evolution driven by the $V \operatorname{ramp} h_{s}(t)$ and of an oscillation driven by the incident surface wave, $H(t) \cos (\Omega t)$, where the slow evolution of $H$ is due to the slow change of the 


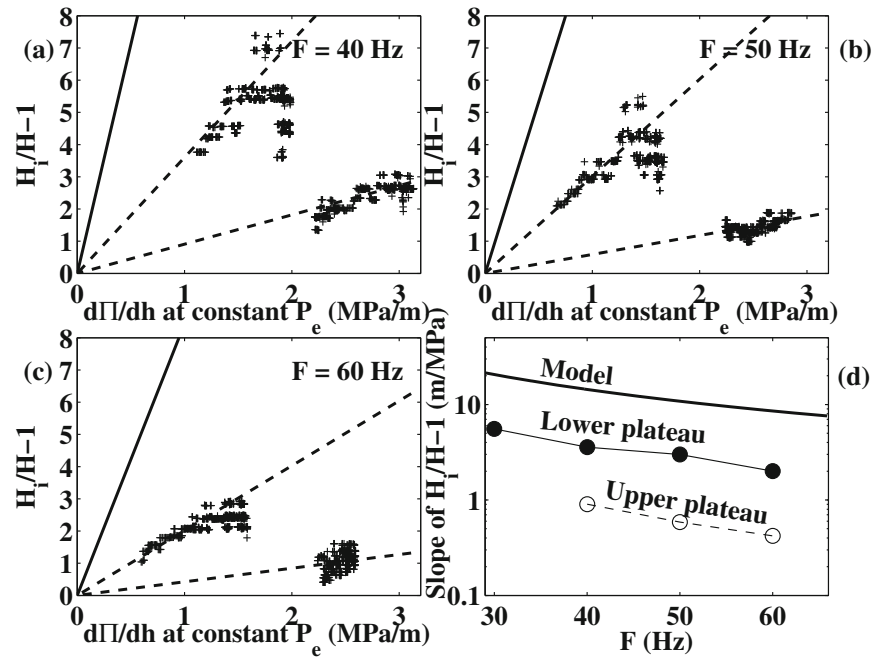

Fig. 5. (a-c) Symbols: variations of the surface wave amplitude ratio $H_{i} / H$ versus the acoustic spring stiffness $-\left.\frac{\partial \Pi}{\partial h}\right|_{P_{e}}\left(h_{s}\right)$ for several values of the surface wave frequency $F$. The dashed lines are best linear fits of the linear parts of each $H_{i} / H$ $\left(-\left.\frac{\partial \Pi}{\partial h}\right|_{P_{e}}\left(h_{s}\right)\right)$ data clusters, the upper (respectively, lower) cluster corresponding to the lower (respectively, upper) plateau of the hysteresis cycle. Solid line: eq. (4). (d) Variation of the slope of the best linear fits of the linear parts of each $H_{i} / H$ $\left(-\left.\frac{\partial \Pi}{\partial h}\right|_{P_{e}}\left(h_{s}\right)\right)$ data cluster versus the surface wave frequency $F$. Solid curve: eq. (4). At those frequencies the wavelength of the gravito-capillary wave is typically $\Lambda=6 \mathrm{~mm}$.

surface effective stiffness during the $V$-ramp. In the presence of the surface wave, the jump of the cavity length toward a more stable average height $h_{s}$ is shown to occur earlier than in the absence of surface wave, as a consequence of the finite value of the oscillation amplitude of the hump height. Consequently, small values of the acoustic spring effect cannot be investigated using this protocol.

Figure 5a-c displays the variation of the measured surface wave amplitude ratio $H_{i} / H$ versus the acoustic spring stiffness $-\left.\frac{\partial \Pi}{\partial h}\right|_{P_{e}}\left(h_{s}\right)$ for several values of the surface wave frequency $F$, together with its prediction using eq. (4). The acoustic spring stiffness is calculated by differentiating with respect to $h_{s}$ the best fits using eq. (3) of the experimental data of fig. 3. This comparison calls for several comments. First, as predicted, $H_{i} / H$ is observed to globally increase linearly with the acoustic spring stiffness. Second, for each frequency, the data exhibit two clusters corresponding to the two plateaux of the hysteresis cycle stridden by $h_{s}(t)$ shown in the inset of fig. 2, the upper (respectively, lower) cluster corresponding to the lower (respectively, upper) plateau. This plateau-dependent value of the acoustic spring indicates that, unexpectedly, its value and/or its effect on the wave propagation actually depends on $h_{s}$ and not only on the acoustic spring stiffness. Moreover, the measured acoustic spring stiffness is found to be systematically weaker than its prediction. This may be ascribed to the roughness of our description of the propagation of the incident surface wave on the hump. The acoustic spring stiffness may also be over- estimated by our model because $H_{i} / H$ is predicted by assuming the hump stiffness as constant over the hump and equal to its maximum value attained at the hump tip. Finally, for the larger values of the acoustic spring encountered along the lower plateau, we observe an unexplained decrease of $H_{i} / H$. The variations of the slope of the linear part of all the $H_{i} / H\left(-\left.\frac{\partial \Pi}{\partial h}\right|_{P_{e}}\left(h_{s}\right)\right)$ clusters versus $F$ are shown in fig. 5 d together with its prediction using eq. (4). As theoretically predicted, the efficiency of the wave amplitude reduction by the acoustic spring effect is observed to decrease when $F$ increases at a rate in agreement with our predictions. To conclude this section, both the observed linear variation of $H_{i} / H$ versus the acoustic spring stiffness and the decrease of the associated slope with the surface wave frequency tend to demonstrate that the acoustic spring effect is actually the cause of the reduction of the surface wave amplitude on the hump. One may however ask whether the incident surface wave could noticeably be scattered by the finite curvature perturbation associated to the hump alone, this process being hence the main cause of the observed reduction of the surface wave amplitude on the hump. As a matter of fact, eq. (4) is based on the linearization of the surface curvature $\kappa\left(h_{s}(r)+\delta h(x, t)\right)=\kappa_{s}(r)+\Delta(\delta h)(x, t)$ ( $\Delta$ is Laplacian operator), which is valid for small values of the surface slope only. Since the characteristic diameter of the hump is $2 w \simeq 1 \mathrm{~mm}$ and its typical height is $1 \mathrm{~mm}$, this linearization may be oversimplifying. Deviations from the above presented linear model are expected to cause the scattering of the incident wave by the curvature field associated to the hump. The experimental test of this hypothesis is presented in the following section.

\section{Wave scattering by the hump}

The technique used to detect the wave scattered by the hump is the following. A parallel beam of white light illuminates the water surface around the hump at almost normal incidence. The reflected beam is directed toward the camera, which images the water surface, see fig. 6a. A picture of the surface is shown in fig. $6 \mathrm{~b}$, where the hump is seen as a dark spot on the right-hand side of the picture of the surface. The crests (respectively, troughs) of the incident surface wave de-focus (respectively, focus) the light reflected by the surface, resulting in dark (respectively, bright) stripes in fig. $6 \mathrm{~b}$ which propagate from the left to the right of the picture. Although the amplitude of the wave backscattered by the hump is so small compared to that of the incident wave that is is barely visible, it can be detected by summing the successive pictures of the surface captured at $1000 \mathrm{fps}$ over one period of the wave. This corresponds to determine the sliding-window time average of the picture of the surface. In the absence of hump, this sliding-window time-averaged picture of the surface is characterized by a homogeneous light distribution. In the presence of hump, the backscattered wave interferes with the incident wave and produces an inhomogeneous, stationary wave of small amplitude compared to the incident wave. But since a stationary wave is characterized 
(a)
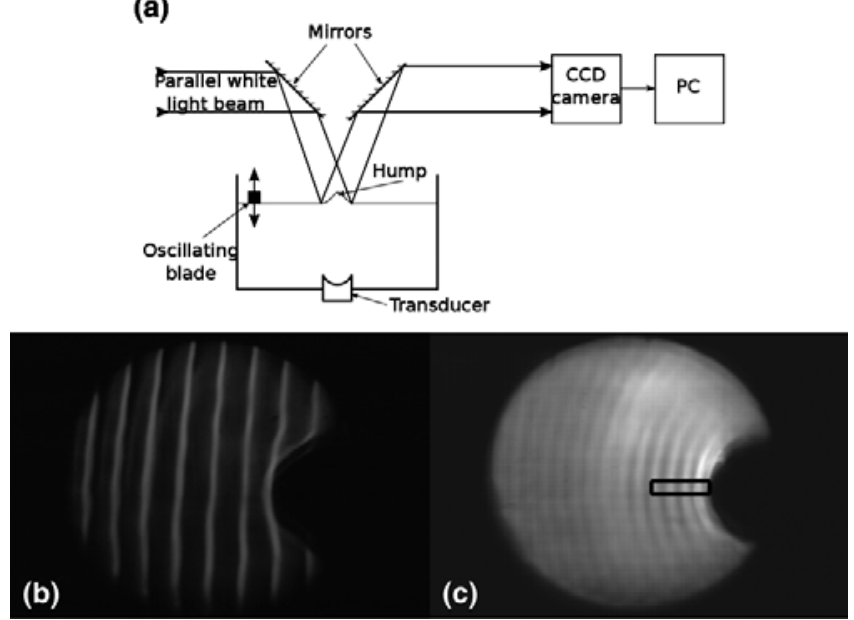

Fig. 6. (a) Experimental setup used to image the backscattered surface wave. (b) Instantaneous picture of the surface imaged by the camera in the presence of incident surface wave. (c) Time-averaged picture of the surface revealing the stationary wave resulting from the interference between the incident wave and the wave backscattered by the hump. The black frame indicates the small area in which the amplitude of the stationary wave is measured

by non-propagating, alternating crests and troughs distant of $\Lambda / 2$, and since the dark stripes produced by the crests are less contrasted than the bright stripes produced by the troughs, as shown in fig. $6 \mathrm{~b}$, the sliding time-averaged picture of the surface displays a $\Lambda / 2$-periodic pattern, which is visible in fig. $6 \mathrm{c}$, and whose contrast increases with the amplitude of the stationary wave. Since the backscattered wave has a cylindrical symmetry, the amplitude of the stationary wave rapidly decreases with the distance to the hump. This is why we measured the contrast of the periodic pattern in a small area of the picture located close to the hump, see fig. $6 \mathrm{c}$.

The variation of this contrast versus time is measured during an up-and-down $V$-ramp striding only the lower plateau of a hysteresis. According to the bistability model presented above and given the chosen experimental conditions, during the $V$ up-ramp the hump height is theoretically predicted to monotonously increase, as shown in fig. 7a, while the acoustic spring is predicted to decrease, as shown in fig. $7 \mathrm{~b}$. Moreover, since the hysteresis cycle is not stridden in this experiment, both quantities are predicted to follow a reversed evolution during the $V$ down-ramp. Consequently, if the incident wave is scattered mainly by the curvature field of the hump, during the up-ramp (respectively, down-ramp) we expect to observe an increase (respectively, decrease) of the amplitude of the scattered wave, hence of the contrast of the periodic pattern appearing on the sliding time-averaged picture of the surface. Conversely, if the incident wave is scattered mainly by the acoustic spring effect, during the up-ramp (respectively, down-ramp) we expect to observe a decrease (respectively, increase) of the amplitude of the scattered wave. The temporal evolution of the contrast of
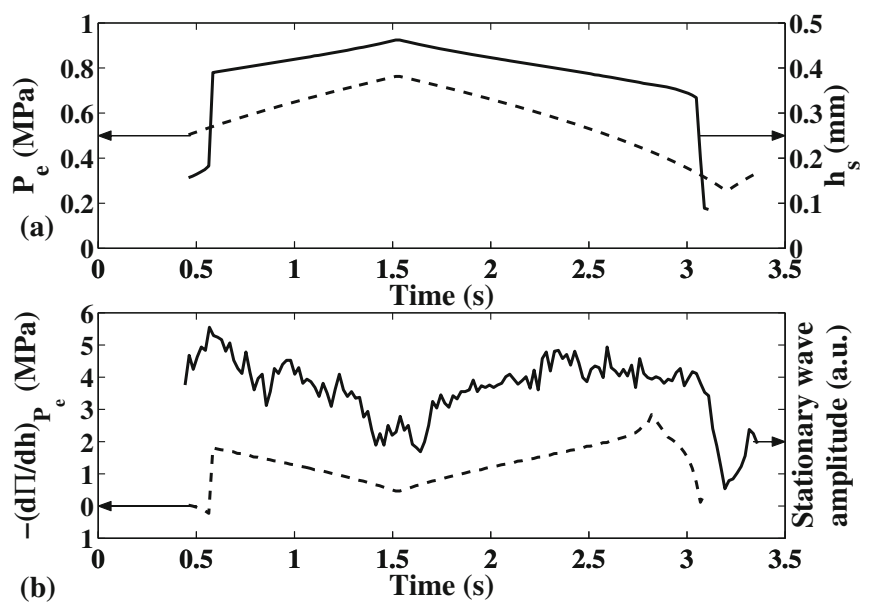

(b)

Fig. 7. (a) Dashed curve: predicted variation versus time of the pressure amplitude $P_{e}$ of the beam emitted by the transducer and entering the cavity during an up-and-down $V$-ramp (scale on the left-hand vertical axis). Solid curve: predicted corresponding variation of the hump height $h_{s}(t)$ (scale on the right-hand vertical axis). (b) Dashed curve: predicted variation of the acoustic spring stiffness $-\left.\frac{\partial \Pi}{\partial h_{s}}\right|_{P_{e}}(t)$ (scale on the left-hand vertical axis). Solid curve: measured contrast of the periodic pattern shown in fig. $6 \mathrm{c}$ resulting from the interference between the incident surface wave and the wave backscattered by the hump.

the periodic pattern is displayed in fig. 7b. It decreases during the up-ramp and displays the reversed evolution during the down-ramp. This indicates that the main cause of the wave scattering is actually the acoustic spring effect and the associated change of the surface impedance on the hump, and that the pressure perturbation associated to the incident surface wave is not noticeably altered by the time-averaged curvature distribution associated to the hump.

\section{Spontaneous hump oscillations}

Spontaneous oscillations of the hump height at a typical frequency of $110 \mathrm{~Hz}$ have also been observed when the liquid surface did not coincide with the transducer focal plane. A typical record of such oscillations during an upand-down $V$-ramp is shown in fig. 8a, and for a constant value of $P_{e}$ in fig. 8b. The intervals in $P_{e}$ and $h_{s}$ over which these oscillations are observed versus the distance between the focal plane and the liquid surface at rest $L_{0}-d_{f}$ are shown in fig. 8c and d, respectively. These oscillations are shown to occur in narrow $P_{e}$ and $h_{s}$ intervals. No oscillation is observed in the range $-2.4 \mathrm{~mm}<L_{0}-d_{f}<0.3 \mathrm{~mm}$. Whereas the mean value of these $h_{s}$ intervals does not exhibit any noticeable dependence on $L_{0}$, the mean value of these $P_{e}$ intervals clearly increases when $\left|L_{0}-d_{f}\right|$ increases. Moreover, the oscillation frequency does not vary by more than $10 \%$ around $110 \mathrm{~Hz}$. Finally, we noticed that these oscillations could not be observed when using a transducer having a central hole such as that used in [25]. 

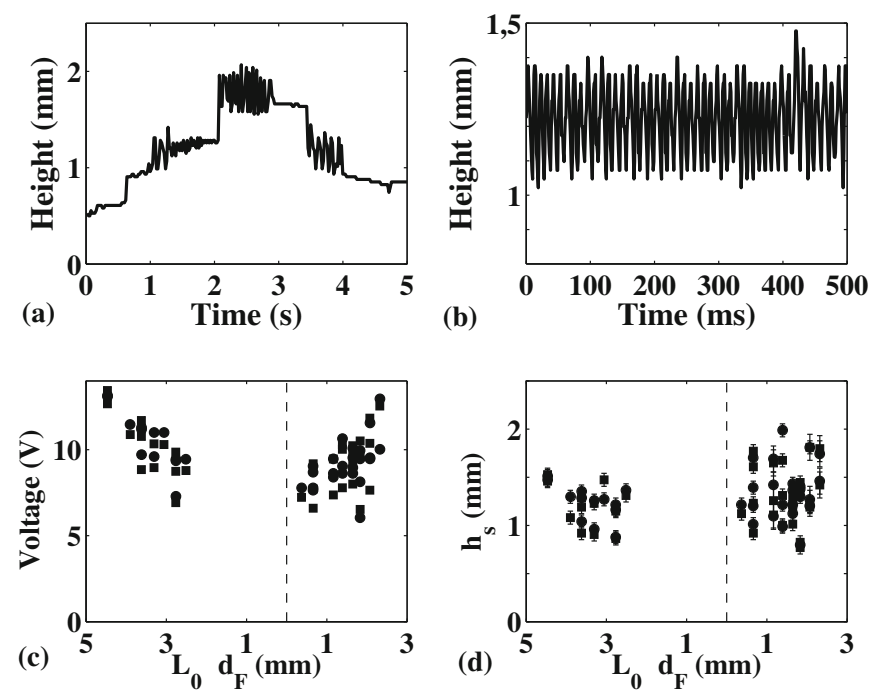

Fig. 8. (a) Time-dependent height of the hump undergoing spontaneous oscillations during an up-and-down $V$-ramp. The liquid surface at rest is $1.4 \mathrm{~mm}$ above the transducer focal plane. (b) Steady-state spontaneous hump height oscillations observed under steady acoustic irradiation at frequency $f=2.5 \mathrm{MHz}$ and pressure oscillation amplitude of the emitted wave $P_{e}=1.8 \mathrm{MPa}$. (c) $P_{e}$ intervals over which these oscillations are observed as a function of the distance between the focal plane and the liquid surface at rest $L_{0}-d_{F}$. (d) Corresponding intervals of time-averaged hump height $h_{s}$ in which these oscillations are observed as function of $L_{0}-d_{F}$.

In order to explain these spontaneous vertical oscillations of the hump, let us consider the effect of a delay of adaptation of the intra-cavity intensity and of the associated radiation pressure exerted on the free surface to a sudden variation of the cavity length on the hump dynamics. In the following, expressions are understood as valid as scaling laws, and their numerical evaluations as orders of magnitude. This delay is equal to the timescale of leakage of the acoustic cavity $\tau=\frac{2 L_{0}}{c\left(1-\left(R R^{\prime}\right)^{2}\right)} \simeq 50 \mu \mathrm{s}$. Considering harmonic oscillations of the cavity length around its equilibrium value $h_{s}$ at frequency $\omega_{\text {osc }} / 2 \pi, \delta h=H \exp i \omega_{\text {osc }} t$, the complex amplitude of the corresponding oscillations of the radiation pressure exerted on the hump tip is $\left.H \frac{\partial \Pi}{\partial h}\right|_{P_{e}}\left(h_{s}\right) \exp (i \phi)$, where $\phi=-\omega_{\text {osc }} \tau<0$. Consequently, the effective stiffness of the hump with halfwidth $w$ is changed into $\sigma w^{-2}+\rho g-\left.\frac{\partial \Pi}{\partial h}\right|_{P_{e}}\left(h_{s}\right) \cos \phi$ and the effective damping of its motion is reduced by an amount proportional to $-\left.\omega_{\text {osc }}^{-1} \frac{\partial \Pi}{\partial h}\right|_{P_{e}}\left(h_{s}\right) \sin \phi[17,27]$. Since the experimentally investigated equilibrium states correspond to negative values of $\left.\frac{\partial \Pi}{\partial h}\right|_{P_{e}}\left(h_{s}\right)$ (see fig. 3), the damping of the hump motion is expected to be reduced, or even possibly becoming negative, leading in this case to spontaneous oscillations at the natural frequency of oscillation of the hump. We indeed note that the observed oscillation frequency agrees well with the value of $200 \mathrm{~Hz}$ deduced from the dispersion equation of surface waves involving the effective stiffness of the hump $f_{\text {osc }}=\left[\left(\sigma w^{-2}+\rho g-\left.\frac{\partial \Pi}{\partial h}\right|_{P_{e}}\left(h_{s}\right) \cos \phi\right) /(\rho w)\right]^{1 / 2} / 2 \pi$, with $\left.\frac{\partial \Pi}{\partial h}\right|_{P_{e}}\left(h_{s}\right) \simeq 1 \mathrm{MPam}^{-1}$ inferred from fig. 5 and $|\phi| \simeq 10^{-2}$. Negative effective damping is indeed wellknown to trigger self-oscillations of opto-mechanical devices [12]. Consequently, this delay may be the cause of the observed vertical oscillations of the hump. It is however difficult to evaluate the natural damping associated to the motion of the hump and hence to determine whether the anti-damping regime has been attained in the present experiment because this natural damping involves viscous dissipation and radiation of surface waves. Moreover, the onset of oscillations has been found to depend on the details of the geometry of the cavity, i.e. the position of the liquid surface relative to the focal plane, the curvature of the hump tip, and the defects of sphericity of the transducer surface, which are not taken into account by our one-dimensional model. But since these oscillations have never been observed when an acoustically transparent interface was involved [28], we believe that they are actually associated to the mutual feedback between the surface deformation and the acoustic cavity.

\section{Conclusion}

We have evidenced the acoustic spring effect in a dynamical configuration by characterizing the reduction of the amplitude of surface waves on the hump induced by acoustic radiation pressure and whose surface stiffness was increased by the mutual feedback between the acoustic field and its height. This demonstrates the universality of the effects of radiation pressure, be it of optical or acoustical origin. Moreover, this study opens the way toward possible applications of the acoustic spring effect to the control of the position, stiffness, and self-oscillations of cantilevers. We have also shown that the acoustic spring effect results in a change of the impedance of the liquid surface, thus inducing surface wave scattering. This shows that the acoustic spring can be used to locally modify the impedance of a liquid surface on demand and hence to induce virtual surface scatterers. This can be compared to the phenomenon of scattering of surface waves by hydrodynamic vortices [29].

\section{References}

1. M. Anderlini, F. Marino, F. Marin, Phys. Rev. D 80, 013001 (2009).

2. V.B. Braginsky, S.E. Strigin, S.P. Vyatchanin, Phys. Lett. A 305, 111 (2002).

3. T. Corbitt et al., Phys. Rev. Lett. 98, 150802 (2007).

4. S.W. Schediwy, C. Zhao, L. Ju, D.G. Blair, P. Willems, Phys. Rev. A 77, 013813 (2008).

5. J. Mertz, O. Marti, J. Mlynek, Appl. Phys. Lett. 62, 2344 (1993).

6. C. Höberger Metzger, K. Karrai, Nature 432, 1002 (2004).

7. K. Kim, S. Lee, J. Appl. Phys. 91, 4715 (2002).

8. M. Vogel, C. Mooser, K. Karrai, R.J. Warburton, Appl. Phys. Lett. 83, 1337 (2003). 
9. A. Schliesser, O. Arcizet, R. Rivière, G. Anetsberger, T.J. Kippenberg, Nat. Phys. 5, 509 (2009).

10. P. Verlot, A. Tavernarakis, T. Briant, P.F. Cohadon, A. Heidmann, Phys. Rev. Lett. 104, 133602 (2010).

11. I. Favero, K. Karrai, Nat. Photonics 3, 201 (2009).

12. O. Arciset, P.F. Cohadon, T. Briant, M. Pinard, A. Heidmann, Nature 444, 71 (2006).

13. M. Hossein-Zadeh, K.J. Vahala, Opt. Lett. 32, 1611 (2007).

14. B.S. Sheard, M.B. Gray, C.M. Mow-Lowry, D.E. McClelland, Phys. Rev. A 69, 051801 (2004).

15. A. Dorsel, J.D. McCullen, P. Meystre, E. Vignes, H. Walther, Phys. Rev. Lett. 51, 1550 (1983).

16. A. Gozzini, F. Maccarone, F. Mango, I. Longo, S. Barbarino, J. Opt. Soc. Am. B 2, 1841 (1985).

17. V.B. Braginskii, A.B. Manukin, Sov. Phys. JETP 25, 653 (1967).

18. V.B. Braginskii, A.B. Manukin, M.Y. Tikhonov, Sov. Phys. JETP 31, 829 (1970).

19. T. Carmon, H. Rokhsari, L. Yang, T.J. Kippenberg, K.J. Vahala, Phys. Rev. Lett. 94, 223902 (2005).
20. F. Marquardt, J.G.E. Harris, S.M. Girvin, Phys. Rev. Lett. 96, 103901 (2006).

21. A. Pai, S.V. Dhurandhar, P. Hello, J.Y. Vinet, Eur. Phys. J. D 8, 333 (2000).

22. T. Carmon, M.C. Cross, K.J. Vahala, Phys. Rev. Lett. 98, 167203 (2007).

23. A.G. Onaran, F.L. Degertekin, Rev. Sci. Instr. 76, 103703 (2005).

24. F.L. Degertekin, B. Hadimioglu, T. Sulchek, C.F. Quate, Appl. Phys. Lett. 78, 1628 (2001).

25. B. Issenmann, R. Wunenburger, S. Manneville, J.P. Delville, Phys. Rev. Lett. 97, 074502 (2006).

26. A. Casner, J.P. Delville, Phys. Rev. Lett. 87, 054503 (2001).

27. C.M. Mow-Lowry et al., Phys. Rev. Lett. 100, 010801 (2008).

28. B. Issenmann, A. Nicolas, R. Wunenburger, S. Manneville, J.P. Delville., EPL 83, 34002 (2008).

29. F. Vivanco, F. Melo, C. Coste, F. Lund, Phys. Rev. Lett. 83, 1966 (1999). 